\title{
Nanotechnology: current uses and future applications in the food industry
}

\author{
Muthu Thiruvengadam ${ }^{1} \cdot$ Govindasamy Rajakumar $^{1} \cdot$ III-Min Chung $^{1}$
}

Received: 24 October 2017 / Accepted: 7 January 2018 / Published online: 13 January 2018

(c) Springer-Verlag GmbH Germany, part of Springer Nature 2018

\begin{abstract}
Recent advances in nanoscience and nanotechnology intend new and innovative applications in the food industry. Nanotechnology exposed to be an efficient method in many fields, particularly the food industry and the area of functional foods. Though as is the circumstance with the growth of any novel food processing technology, food packaging material, or food ingredient, additional studies are needed to demonstrate the potential benefits of nanotechnologies and engineered nanomaterials designed for use in foods without adverse health effects. Nanoemulsions display numerous advantages over conventional emulsions due to the small droplets size they contain: high optical clarity, excellent physical constancy against gravitational partition and droplet accumulation, and improved bioavailability of encapsulated materials, which make them suitable for food applications. Nano-encapsulation is the most significant favorable technologies having the possibility to ensnare bioactive chemicals. This review highlights the applications of current nanotechnology research in food technology and agriculture, including nanoemulsion, nanocomposites, nanosensors, nano-encapsulation, food packaging, and propose future developments in the developing field of agrifood nanotechnology. Also, an overview of nanostructured materials, and their current applications and future perspectives in food science are also presented.
\end{abstract}

Keywords Nanoemulsion $\cdot$ Nanocomposites $\cdot$ Nanosensor $\cdot$ Nano-encapsulation $\cdot$ Food packaging

\section{Introduction}

Nanoscience and nanotechnology are innovative scientific advancements that have been introduced only in this century. Their utilizations in food and agriculture productions are almost modern compared with that of medicine delivery and pharmaceuticals. Nanotechnology has developed as the scientific advancement to grow and transform the entire agrifood area, with the potential to elevate global food production, furthermore to the nutritional value, quality, and safety of food (Sekhon 2014; Chung et al. 2017). Nanotechnology uses in food science are going to influence the most important aspects of food manufacturing from food protection to the molecular synthesis of new food products

Govindasamy Rajakumar

govindr@konkuk.ac.kr

Ill-Min Chung

imcim@konkuk.ac.kr and ingredients (Pathakoti et al. 2017). Nanotechnology is expected to facilitate the following development stage of genetically altered crops, input to the production of animal and fisheries, chemical insecticides and precision farming methods. Precision farming is one of the most important techniques utilized for increasing crop productivity by monitoring environmental variables and applying the targeted action (Chen and Yada 2011). Food endures a variability of post-harvest- and processing-persuaded changes that affect its biological and biochemical maquillage. Thus, nanotechnology development in the areas of biochemistry and biology could also affect the food manufacturing (Sozer and Kokini 2009; Jain et al. 2016). There is a need to develop simpler, faster, more sensitive and low-cost approaches for the observation and quantification of impurities in foods. Within the past decade, with remarkable advances in nanoscience, nanotechnology-enabled sensors and systems have been increasingly used to develop rapid and noninvasive methods of detection of food contaminants.

1 Department of Applied Bioscience, College of Life and Environmental Sciences, Konkuk University, Seoul 143-701, Republic of Korea 


\section{Nanotechnological applications in food industry}

Nanotechnology has been reported as the new industrial revolution, both developed, and developing countries are investing in this technology to secure a market share. At present, the USA leads with a 4-year, 3.7-billion USD investment through its National Nanotechnology Initiative (NNI). The USA is followed by Japan and the European Union, which have both committed substantial funds (750 million and 1.2 billion, including individual country contributions, respectively, per year). Others such as India, South Korea, Iran, and Thailand are also catching up with a focus on applications specific to the economic growth and needs of their countries (Kour et al. 2015). Food processing approaches that involve nanomaterials include integration of nutraceuticals, gelation and viscosifying agents, nutrient propagation, mineral and vitamin fortification, and nano-encapsulation of flavors (Huang et al. 2010). Thus, systems with physical structures in the nanometer distance range could affect features from food safety to molecular synthesis. Nanotechnology may also have the potential to enhance food quality and safety. Many studies are assessing the ability of nanosensors to improve pathogen detection in food systems. Nanofoods are products that were grown processed or packaged with the aid of nanotechnology or materials produced with nanotechnology (Fig. 1). In this review, we discuss some current nanotechnology research in food technology and agriculture, including processing, packaging, nano-additives, cleaning, and sensors for the detection of contaminants, and propose future developments in the developing field of agrifood nanotechnology (Fig. 2).

\section{Nano-delivery of food ingredient}

\section{Nanoemulsion}

The emulsion is two or more combination of liquids (oil/ water system) that do not simply combine. The diameters of nanoemulsion to discrete droplets measure $500 \mathrm{~nm}$ or less. It can contain functional constituents within their droplets, which can ease a decrease in chemical degradation (Ravichandran 2010). The promising vicinity of nanotechnology within the food industry is the usage of nanoemulsions as carriers for lipophilic bioactive constituents, flavoring agents, antioxidants, preservatives, and drugs (Silva et al. 2012). An interest has been developing in the use of nanoemulsions within the food, beverage, and medicinal industries since they have some potential benefits over conventional emulsions for certain applications (Komaiko and McClements 2016). Nanoemulsions are kinetically uniform liquid-in-liquid dispersions with droplet sizes about $100 \mathrm{~nm}$ (Komaiko and McClements 2016). Nanoemulsion-based delivery system can also improve the bioavailability of the encapsulated components due to the small particle size and high surface-to-volume ratio (Sun et al. 2015). As a trendy advice, when used in the food manufacturing nanotechnology needs to be reasonable, easy to utilize, and with willingly perceived benefits in order to be a real another to the normal techniques. There are diverse challenges like limited food-grade stabilizers or other ingredients obtainable. The food industry would like to prepare nanoemulsions from legally acceptable, label-friendly, and economically viable ingredients. The most important is the toxicological concerns because the nanosize of the droplets that could alter the normal function of the gastrointestinal tract (Sugumar and Singh 2016). A fascinating food application of essential
Fig. 1 Framework for integrating nanoresearch areas and the food supply chain

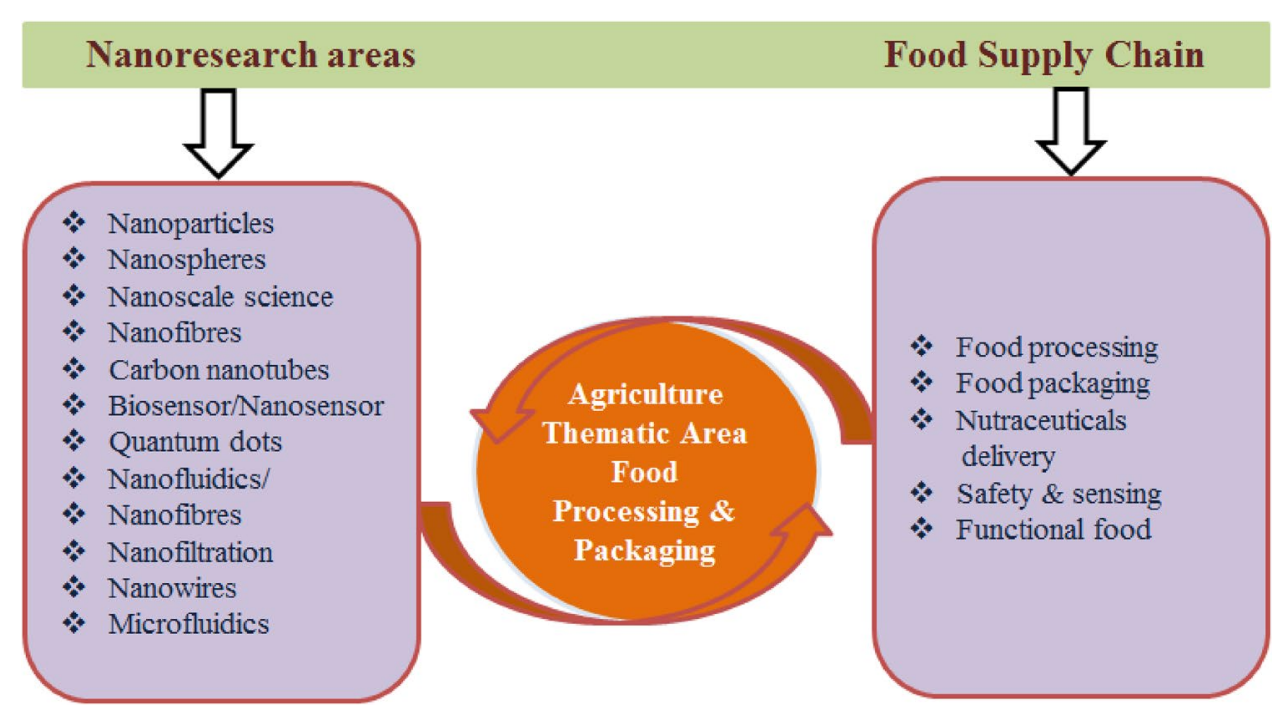




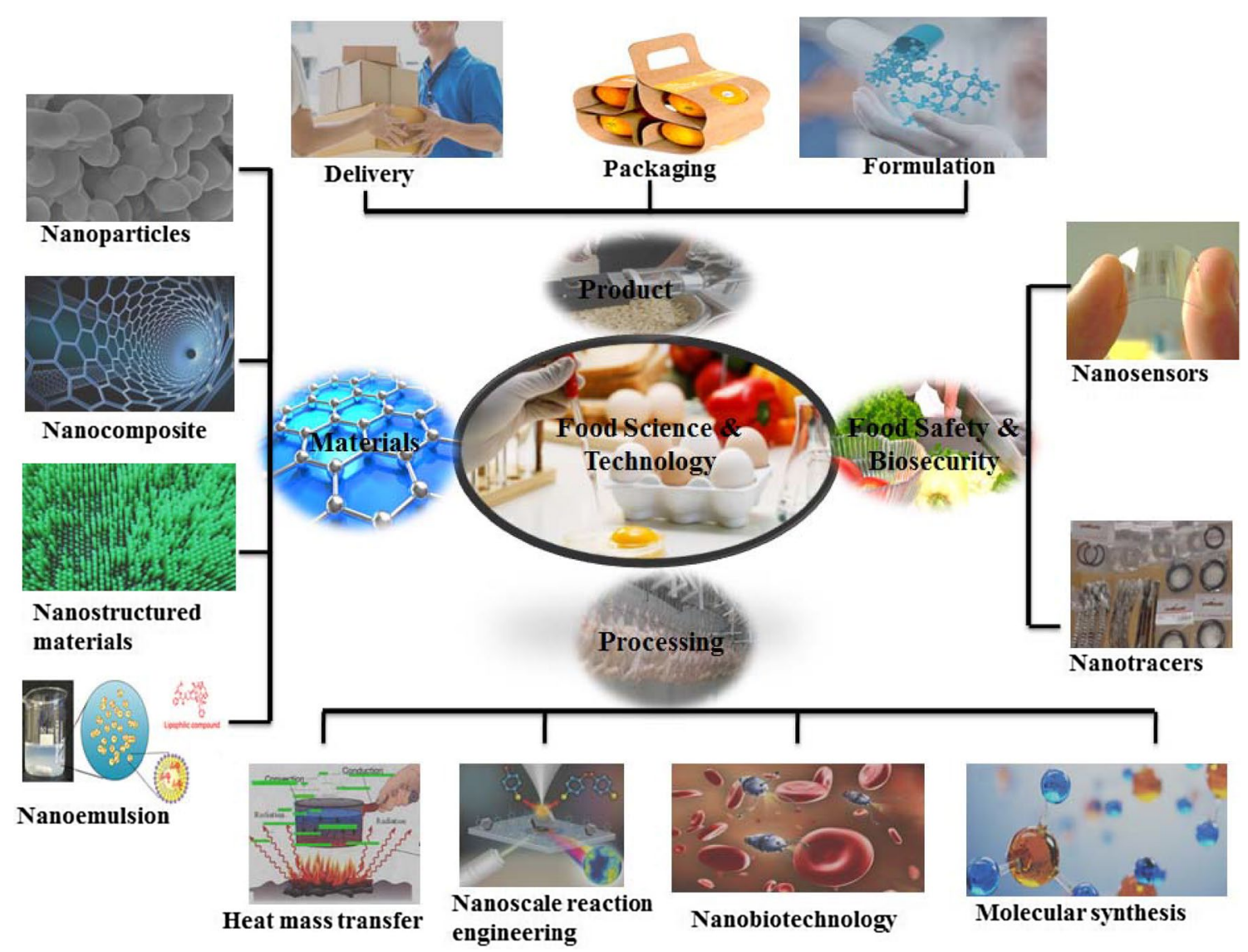

Fig. 2 Different steps of food management that involve several steps (processing, packaging, and preservation) and these aided by nanotechnology with the assistance of several nanomaterials

oils nanoemulsion has been observed in plums. Recently, lemongrass oil nanoemulsion was used to evaluate antimicrobial properties, physical, and chemical changes in plums (Kim et al. 2013). The nanoemulsion was able to inhibit E. coli and Salmonella population without altering essence, breakability, and smoothness of the product. It was also able to decrease ethylene production and retard alterations in lightness and concentration of phenolic compounds (Amaral and Bhargava 2015).

Nanoemulsions have some potential benefits over traditional emulsions for specific uses within food and beverage products. Nanoemulsions typically have a better consistency about particle accumulation and gravitational separation (Komaiko and McClements 2016). Nanoemulsions can be assembled through a variety of approaches, which can be classified as low-energy or high-energy methods depending on the inactive principle (Gupta et al. 2016). Various types of nanoemulsions with more complex properties, e.g., nanostructured multilayer emulsions or uncountable emulsions, produce various encapsulating skills from a single delivery system; this can promote the activity of the active components and facilitate their release in response to an activator.
For example, Nestle and Unilever have developed a nanoemulsion-based ice cream with less content of fat (Singh 2015). Nano-encapsulation of food ingredients and additives had been carried out to provide protecting hurdles, taste and flavor masking, controlled release, and better dispensability for water-insoluble food ingredients and additives. There is a developing public concern regarding the toxicity and adverse effect of nanoparticles on human health and environment (Cushen et al. 2012).

Lipid-based nanoemulsions are better for the delivery of constituents within biological systems than traditional nanoemulsions. However, the high lipid content of these nanoemulsions results in adverse effects on the body, such as obesity and cardiovascular diseases (Pradhan et al. 2015). Some approaches for forming nanoemulsions using lowenergy methods require the presence of cosolvents (e.g., polyols, such as propylene glycol, glycerol, and sorbitol) or cosurfactants (e.g., short and medium-chain alcohols) (McClements and Rao 2011). Nanoemulsions present numerous benefits such as cleansing of equipment and high clearness without compromising product presence and flavor (Fig. 3). Nano-sized functional molecules that

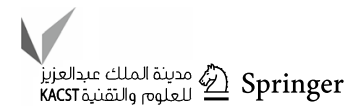




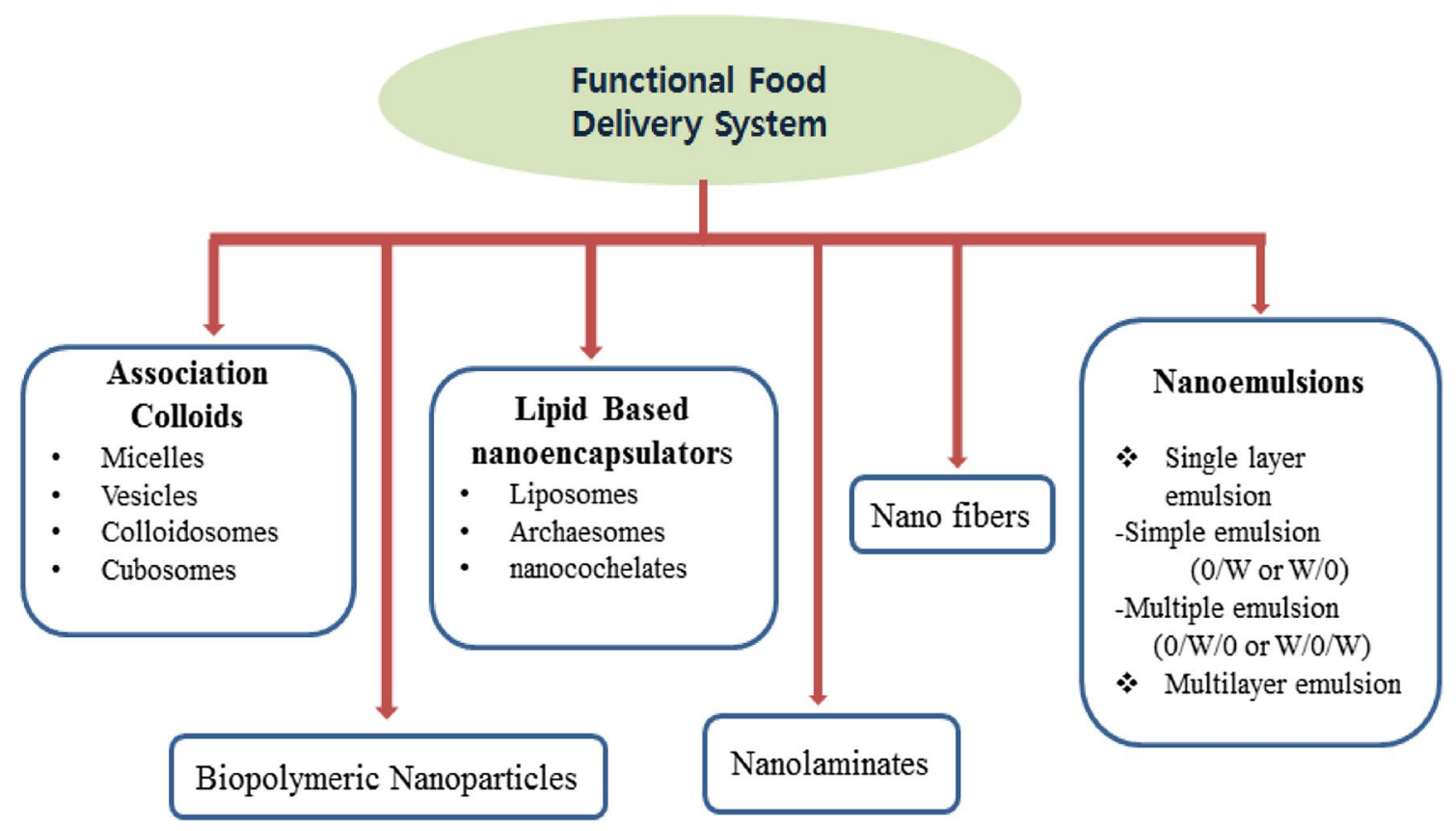

Fig. 3 Nanofunctional food delivery systems

are encapsulated by the self-assembled nanoemulsions are used for targeted delivery of lutein; $\beta$-carotene; lycopene; vitamins A, D, and E3; co-enzyme Q10; and omega-3-fatty acids (Choi et al. 2011). The use of nanoemulsions to food systems still poses challenges that need to be addressed both concerning the production process, particularly their price and of the characterization of both the resultant nanoemulsions and the food systems to which they will be applied to product safety and acceptance. Nanoemulsions exhibit numerous benefits over traditional emulsions because of their small droplet dimensions: high optical clearness, excellent physical constancy against gravitational partition and droplet accumulation, and improved bioavailability of encapsulated materials, which make them suitable for food applications (Oca-Avalos et al. 2017).

\section{Nano-encapsulation}

Nanotechnology can also facilitate encapsulation of drugs or other components for protection against environmental factors and can be used in the plan of food ingredients, e.g., flavors and antioxidants (Ravichandran 2010). Micro-encapsulation is used to increase bioavailability, control release kinetics, minimize drug side effects, and cover the bitter taste of medicinal substances in the pharmaceutical industry. In the food industry, nanoemulsions are used in the organized release of additives and the manufacturing of foods containing functional constituents, such as probiotics and bioactive ingredients (Kuang et al. 2010). Currently, numerous techniques of nano-encapsulation are progressively rising with their own merits and demerits. Techniques including emulsification, coacervation, inclusion complexation, nanoprecipitation, solvent evaporation, and supercritical fluid technique are enduring techniques for nano-encapsulation of food substances. Moreover, solvent evaporation and nanoprecipitation remain to be particular techniques for encapsulation of lipophilic bioactive compounds. However, all the encapsulation technologies, in the long run, depend on proper drying strategies to provide nanoencapsulates in powder form. Lee et al. (2017) conducted a study to improve the water solubility and antimicrobial activity of milk thistle silymarin by nano-encapsulation and to assess the functions of silymarin nanoparticle-containing film as an antimicrobial food-packaging agent. Further, the author stated that the incorporation of silymarin in WCS/-PGA nanoparticles could be an effective approach for improving the solubility and the antimicrobial activity of silymarin. Biodegradable films containing silymarin nanoparticles could efficiently control the growth of food microorganisms. Nano-encapsulation of valuable microorganisms, e.g., probiotics, is advantageous because targeted and site-specific delivery to the desired region of the gastrointestinal tract can be achieved. These nano-encapsulated designer bacterial preparations can be used in vaccine preparation and to enhance the immune response (Vidhyalakshmi et al. 2009). Additionally, nanoemulsions have been shown to improve the health benefits of curcumin (Wang et al. 2008). Most nanoencapsulates have shown excellent bioavailability, and few encapsulates have reported good inhibitory effect against certain targeted diseases. However, presently, the possible risks of 
nanomaterials to human fitness are unknown and need to be explored and studied (Ezhilarasi et al. 2013). Moreover, the regulatory issues on nanofoods are still being developed, and it is expected that national bodies will increase initiatives to control, administrate, and promote the proper development of nano-sized food-related products.

\section{Packaging of food items}

\section{Nanocomposites}

Nanocomposites are mostly exploited in the area of food packaging, as they are eco-friendly and biodegradable. Nanocomposites exhibit extremely multipurpose chemical functionality and are therefore used for the growth of high obstacle properties (Pandey et al. 2013). A nanocomposite-based commercialized fertilizer, Guard IN Fresh, helps fruits and vegetables to ripen by scavenging ethylene gas (Gupta and Moulik 2008). Nanoclays are made of aluminum silicates, commonly mentioned to as phyllosilicates, and are low-cost, constant, and eco-friendly (Davis et al. 2013). The nanocomposite is a multiphase material resulted from the combination of two or more constituents, containing a continuous phase (matrix) and a discontinuous nano-dimensional phase with at least one nano-sized dimension (with less than $100 \mathrm{~nm}$ ). The development of bio-nanocomposite materials for food packaging is significant not only to reduce the environmental problem, but also to improve the functions of the food packaging materials (Othman 2014). Moreover, nanoparticles could impart as their active or intelligent properties to food packaging so that they can preserve the food against external factors and increase the food's stability through antimicrobial properties and/or responding to environmental changes. In spite of several advantages of nanomaterials, their use in food packaging may cause safety problems to human health since they exhibit different physicochemical properties from their macro-scale chemical counterparts (Hanarvar 2016). The usage of nanocomposites for food packaging defends not only food, but also develops the shelf-life of food products and overcomes environmental problems associated with the use of plastics. Most packaging materials are not degradable, and popular biodegradable films have a poor barrier and mechanical properties; therefore, these properties must be significantly improved before these films can replace conventional plastics and help to manage universal waste problems (Sorrentino et al. 2007).

Shankar and Rhim (2016) produced nanocomposite films including PBAT (polybutylene adipate-co-terephthalate) and silver nanoparticles. The maximum plasmonic absorption of silver nanoparticles was detected at $435 \mathrm{~nm}$. Moreover, the dramatic increase in tensile strength and water vapor permeability of the film was attributed to the presence of silver nanoparticles. Altogether, the formulated nanocomposite presented important features to be applied in packaging materials due to their UV-screening and biocidal activities. In addition to the abovementioned benefits, nanomaterials have also been developed continuously to enhance the physical and mechanical properties of packaging in terms of tensile strength, rigidity, gas permeability, water resistance and flame resistance. Aimed at providing those properties above, polymer nanocomposites are the latest materials with an enormous potential for use in the active food packaging industry (Youssef 2013). Better use of polymer-nanocomposite in the industry in Europe is going very slowly. The main reasons are the cost price of materials and processing, restrictions due to legislation, acceptance by customers in the market, lack of knowledge about the effectiveness and influence of nanoparticles on the ecological and on human health. The potential risk due to the migration of nanoparticles in food, and balance between the use of biomass for the production of foods (Bratovčić et al. 2015). Polymer nanocomposite-based food packaging material with antimicrobial properties is particularly useful due to the high surface-to-volume ratio of nanofillers. In addition, this property increases the surface reactivity of the nano-sized antimicrobial agents compared to the bulk counterpart, making them able to kill microorganisms. The performance properties, for example, mechanical, barrier, thermal, optical, biodegradation, and antimicrobial properties are found in polymer nanocomposites for the packaging applications (Fig. 3).

\section{Nanosensors}

Nanosensors in conjunction with polymers are used to screen food pathogens and chemicals during storage and transit processes in smart packaging. Additionally, smart packaging confirms the integrity of the food package and authenticity of the food product (Pathakoti et al. 2017). Nano-gas sensors, nano-smart dust can be used to detect environmental pollution (Biswal et al. 2012). These sensors are composed of compact wireless sensors and transponders. Nanobarcodes are also an efficient mechanism for detection of the quality of agricultural fields (Sonkaria et al. 2012). An electrochemical glucose biosensor was nanofabricated by layerby-layer self-assembly of polyelectrolyte for detection and quantification of glucose (Rivas et al. 2006). Nanosensors can detect environmental changes, for example, temperature, humidity, and gas composition, as well as metabolites from microbial growth and byproducts from food degradation (Fig. 4). The types of nanosensors used for this purpose include array biosensors, carbon nanotube-based sensors, electronic tongue or nose, microfluidic devices, and nanoelectromechanical systems technology (Sozer and Kokini 2009). Polymer nanocomposites from carbon black and polyaniline to detect and identify foodborne pathogens (Bacillus cereus, Vibrio parahaemolyticus, and Salmonella spp.)

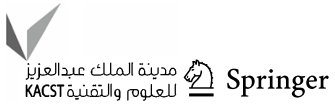


Fig. 4 Different types of nanosensors and examples of their use in the food sector

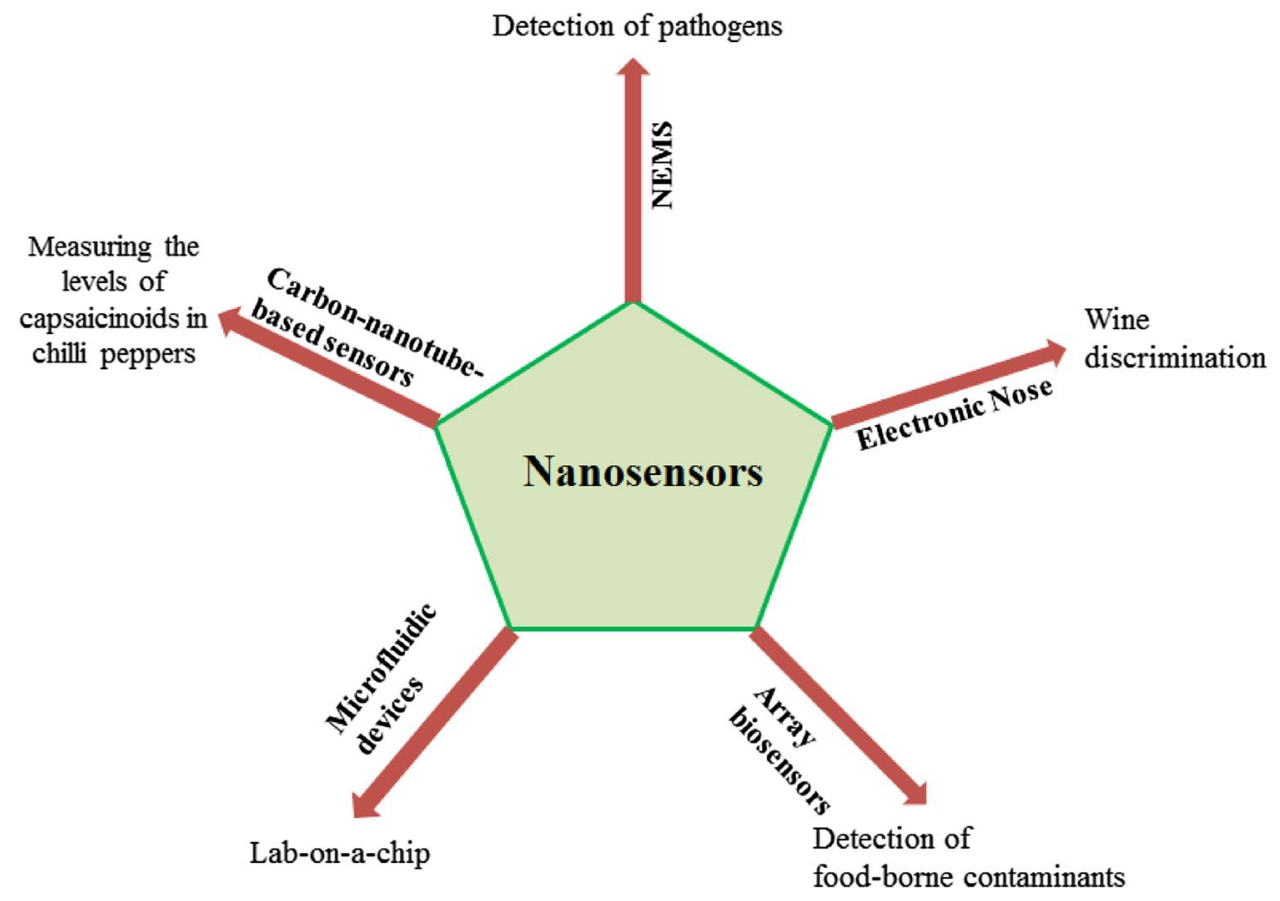

based on the specific response patterns for each microorganism, as triggered by different vapors produced during their metabolism (Arshak et al. 2007). A liposome-containing nanosensor based on microfluidics showed that the main benefit of microfluidic sensors is their simple arrangement and their capability to identify constituents of interest fast in only microliters $(\mu \mathrm{L})$ of sample volume (Sozer and Kokini 2009). The combination of nanosensors into food packaging has shown in various benefits than traditional sensors for example speed of analysis, enhanced sensitivity, specificity and multiplex systems (sample throughput), reduced cost and assay complexity (Singh et al. 2017). The sensors based on nanomaterials (nanosensor), both chemical sensors (chemical nanosensors) and biosensors (nanobiosensors), can be used online and combined into existing industrial process and distribution line or off-line as speedy, simple, and transportable, as well as disposable, sensors for food contaminants (Kuswandi 2017).

Nanosensors can also be used to determine the qualities of various foods, including wine, coffee, juice, and milk. The sensors are designed using layer-by-layer macromolecule ultra-thin films that show increases in surface area and 10,000-fold higher sensitivity than the human tongue. Nanosensors can further be fixed to packaging to identify microorganisms contaminating food. The packaged food product does not need to be directed to the laboratory for sampling; instead, the sensors indicate the food quality and can be directly interpreted by consumers based on color changes. Sensors that are typically used sensors in food packaging are gas detectors and time-temperature indicators, including array biosensors, nanoparticles in solution, nanoparticle-based sensors, nano-test strips, electronic noses, and nanocantilevers (Tang et al. 2009). The use of nanoparticles to develop nanosensors for detection of food contaminant and pathogens in the food method is another possible use of nanotechnology. Indeed, tailor-made nanosensors for food analysis, flavors or colors, drinking water and clinical diagnostics have been developed ( $\mathrm{Li}$ and Sheng 2014). Nanosensors have also been applied for detection of organophosphates in plants, fruits, and water. Owing to the high water solubility, toxicity, and extensive use of pesticides in agriculture, there is an urgent requirement for highly sensitive and selective analytical systems for residue analysis of these pollutants (Valdés et al. 2009). Advances in nanosensor technology were discussed in a recent review highlighting magnetic immune sensors based on biomolecules connected with gold nanoparticles with a broad range of uses in food (Vidotti et al. 2011). An SPR-based biosensor was applied for fast identification of Campylobacter jejuni in samples of broiler chickens, and the specificity and sensitivity of distribution antibodies against $C$. jejuni were tested with Campylobacter and non-Campylobacter bacterial strains. Nanosensors and nano-based smart delivery methods are the uses of nanotechnology that are presently working in the agricultural production to help with fighting viruses and other crop pathogens, as well as to boost the effectiveness of agrochemicals at lower amount proportions (Mousavi and Rezaei 2011). Jebel and Almasi (2016) analyzed the antibacterial effect of $\mathrm{ZnO}$ nanoparticles embedded in cellulose films (impacts on E. coli and S. aureus). They also applied ultrasound treatment to the bacteria and observed remarkable antibacterial performance. 
Zhao et al. (2011) created a rapid, sensitive DNA strip sensor based on gold nanoparticle-labeled oligonucleotide probes to detect Acidovorax avenae subsp. citrulli. Both qualitative and semiquantitative findings of the target DNA were obtained; the qualitative limit of detection of the strip sensor was $4 \mathrm{nM}$. Oxonica Inc. (USA) developed nanobarcodes for use with dessert items or pellets to be delivered using an altered microscope for anti-counterfeiting determinations. The additional trend in the use of nano-packaging is nano-biodegradable packaging. The usage of nanomaterials to develop bioplastics may allow bioplastics to be used as a replacement for fossil fuel-based plastics for food packaging and carry bags. These devices have been receiving growing attention because the need for detecting and measuring at the molecular, physical and chemical properties of toxins, pollutants, and analytes in general (Table 1) (Guo et al. 2015; Martínez-Bueno et al. 2017). Li and Sheng (2014) reported the applications of gold nanoparticles and CNTs in food contamination detection. Potential research focus has also been suggested. Nanosensors developed based on the molecularly imprinted polymer technology include those used for the detection of trypsin, glucose, catechol, and ascorbic acid (Pathakoti et al. 2017). For human health, nanotechnology has tremendous interest in food detection and will be receiving more and more attention shortly. The food industry is eager to benefit from its revolutionary discovery as much as possible. The purpose of research and development of nanotechnology is to realize the efficient control of the microscopic world. Taking advantage of nanotechnology, researchers are beginning to realize the promising future in the field of biological sensors in food detection.

\section{Food packaging}

The biodegradability of a packaging material can be augmented by integrating inorganic elements, for example, mud, into the biopolymeric medium and can be measured with surfactants that are utilized for the alteration of the layered silicate. The use of inorganic elements also makes it possible for food packaging to have multiple functionalities, which could aid in the development of methods to deliver fragile micronutrients within edible capsules (Sorrentino et al. 2007). Food packaging is thought to be the main application of nanotechnology in the food industry. The adding of nanoparticles to shaped substances and films has been demonstrated to increase the properties of these materials, mainly durability, temperature resistance, flame resistance, barrier properties, optical properties, and recycling properties. Nano-packaging can also be designed to release enzymes, flavors, antimicrobials, antioxidants, and nutraceuticals to extend shelf-life (Cha and Chinnan 2004). Giannakas et al. (2016) have reported that addition of nanoclays is inducing the antimicrobial properties of $\mathrm{PVOH} /$ chitosan films and increases antimicrobial activity up to $44 \%$ for NaMMT and up to 53\% for OrgMMT. Antimicrobial nanomaterials present an amount of current packaging concept planned to bring the vigorous nanoparticles that can be combined into a food package (Mihindukulasuriya and Lim 2014). Nanotechnology uses in the food manufacturing can be exploited to produce stronger tastes and color quality or detect bacteria in packaging, and safety by growing the obstacle properties and holds great potential to offer benefits not just within food products, but also around food products. In fact, nanotechnology introduces new chances for innovation in the food industry fast, but uncertainty and health concerns are also emergent (Sekhon 2014).

\section{Benefits of nanomaterials in food packaging uses}

Bioactive-packaging materials can aid the oxidation of foodstuffs and avoid the development of off-flavors and unwanted textures. Nonsustainable production, lack of recyclability, and insufficient mechanical and barrier properties are some of the ongoing challenges faced by the food and packaging industries. Although metal and glass are excellent barrier materials that can be used to inhibit undesirable mass transport in food packaging, plastics are still popular due to their lightweight, formability, cost effectiveness, and versatility. Indeed, the packaging industry accounts for more than $40 \%$ of all plastic usage, with half of this $40 \%$ used for food packaging (Rhim et al. 2013). Ravichandran (2010) revealed that the development of exciting novel nanotechnology products for food packaging, and some antimicrobial films had been introduced to increase the shelf-life of food and dairy products (Fig. 5). Moreover, food preservation and food packaging materials have become essential in the food industry. Food spoilage can be detected using nanosensors; thousands of nanoparticles fluoresce in several colors after coming into contact with food pathogens. In our studies of the significance of time in nourishment microbiology, the chief goal of nanosensors was to decrease the time for pathogen detection from days to hours or even minutes (Bhattacharya et al. 2007). Packaging prepared with nanosensors can also track either the internal or external circumstances of food products, vessels, and pellets. For example, Opel, which is used to make Opalfilm, containing 50-nm carbon black nanoparticles, was used as a biosensor that could change color in response to food spoilage.

Bioactive packaging resources necessity to be prepared to maintain bioactive chemicals, for example, probiotics, prebiotics, bioavailable flavonoids, and encapsulated vitamins, under optimal conditions, till they are released in a controlled method into the nourishment product (López-Rubio et al. 2006). Carbon nanotubes, which are mostly used as packaging for foods, constantly migrate into foods and can be used to control toxicity on the skin and lungs of human (Mills and 


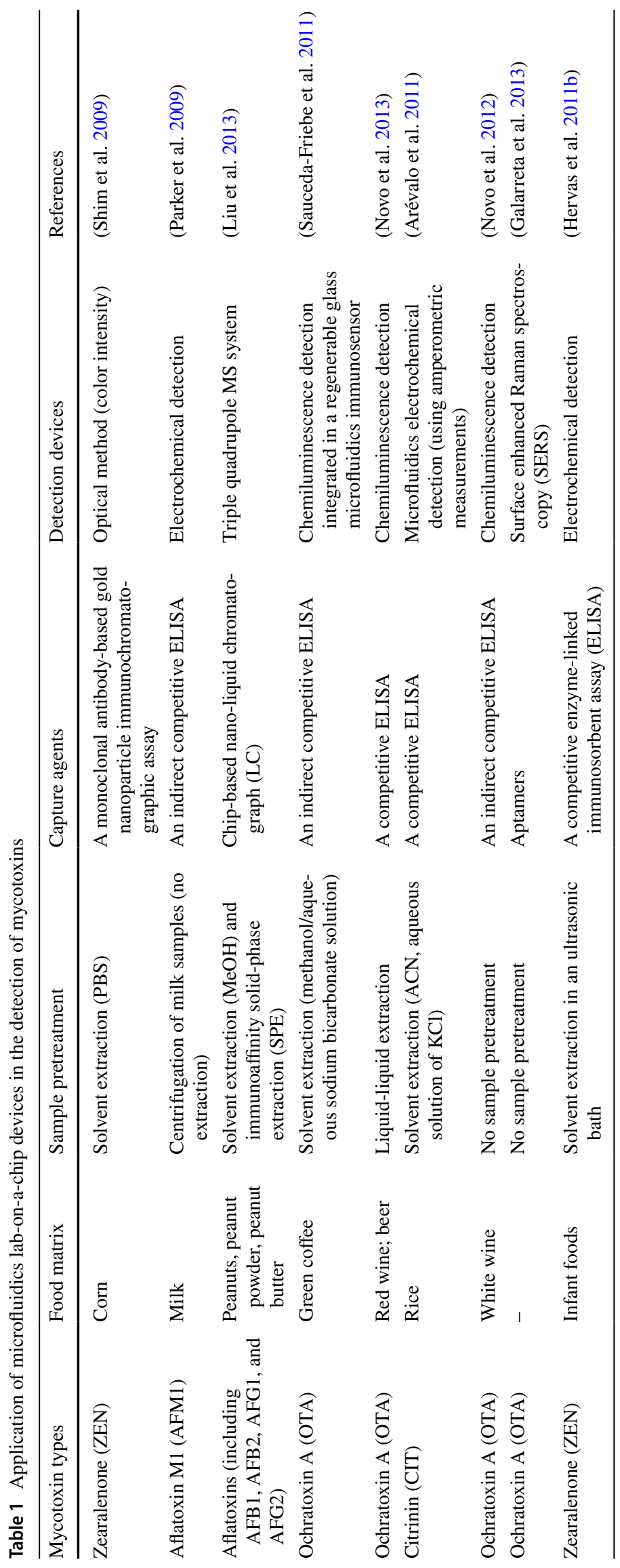


Fig. 5 Benefits and risks of nanotechnology applications in food and related products

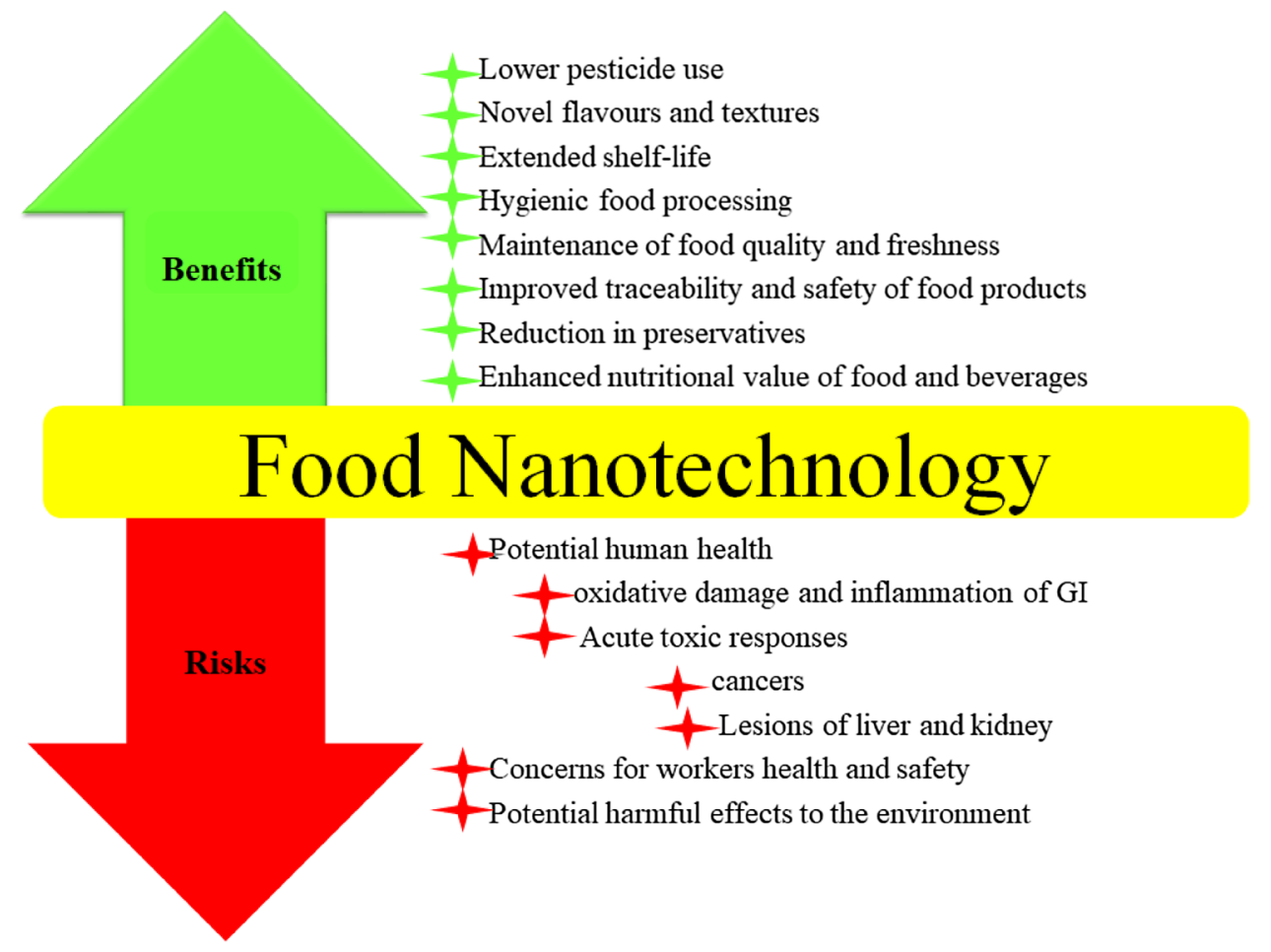

Hazafy 2009). Lemes et al. (2008) prepared a nanocomposite with multiwalled carbon nanotubes and the biopolymer poly(3-hydroxybutyrate-co-3-hydroxyvalerate), enhancing its mechanical properties. Several microorganisms produce this polymer as reserve materials, and its use as packaging in food was approved in Europe. Reynolds (2007) demonstrated that approximately 400-500 nano-packaging products are commercially available, and nanotechnology is expected to be utilized in the manufacturing of $25 \%$ of all food packaging within the next generation. An ingestible nano-based track and trace technology was developed by pSiNutria, a division of the nanotechnology company pSivida. Possible pSiNutria products include products to identify pathogens in food for food tracing and preservation and temperature measurements in food storage (Alfadul and Elneshwy 2010). The FDA controls nanofoods, and the maximum allowable amounts of nanomaterials in food packaging and organic chemicals are monitored by the Environmental Protection Agency (EPA) in the USA. Though neither the EPA nor the FDA has documented nanomaterials as novel chemicals or have required any new oversight of these materials-based products to engage in early and frequent consultation with the agency (Badgley and Perfecto 2007).

\section{Application of nanotechnology in foods and bioactives}

Archaeosomes are a type of microbial lipid membrane resistant to oxidation, chemical and enzymatic hydrolysis, low $\mathrm{pH}$, high temperature, and the presence of bile salts due to the hostile living environment of Archaea microbes (Mozafari 2006). Canham (2007) found that the milk protein $\alpha$-lactalbumin in certain conditions can undergo selfassembly to form tubular nanostructures. Such tubes are thousands of nanometers long, their diameter is $20 \mathrm{~nm}$, and the inner cavity diameter is about $8 \mathrm{~nm}$. These structures are formed in several stages. In the first stage, $\alpha$-lactalbumin is partially hydrolyzed through the activity of a protease from Bacillus licheniformis. Also, along with other components, several derivatives with molecular masses varying from 10 to $14 \mathrm{kDa}$ are formed. In the presence of calcium ions, this mixture self-assembles into helical tubes. Nanocochleates resulting from soy and calcium have been found to be suitable for the nano-encapsulation of vitamins, omega-3 fatty acids, and lycopene without affecting the organoleptic properties of foods (Joseph and Morrison 2006). Dairy products, beverages cereals, and bread are now supplemented with minerals, vitamins, bioactive peptides, probiotics, plant sterols, and antioxidants. Some of these active components are being added to foods as nanoparticles or particles of a few hundred nm in size (Shelke 2008). Gupta and Gupta (2005) demonstrated that nanometer-sized particles could be produced using food-grade biopolymers, e.g., polysaccharides or proteins, by inducing phase separation in mixed biopolymer systems, self-association, or aggregation. Nanoparticles are added to various foods to increase flow properties, color, and stability during processing, or shelf-life. For example, aluminosilicate materials are typically used as anticaking

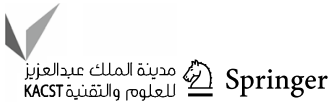


agents in powdered processed foods, whereas anatase titanium dioxide is a normal food whitener and brightener additive employed in sweets, some cheeses, and sauces (Ashwood et al. 2007). The applications explored here were particularly chosen because they are the most likely nanofood products to be accepted by consumers in the short term. Thus, food nanotechnology is still young, and the future of this exciting field is still largely uncertain. Regardless of how applications of nanotechnology in the food sector are ultimately marketed, governed, or perceived by the public, it seems clear that the manipulation of matter on the nanoscale will continue to yield exciting and unforeseen products.

\section{Agriculture}

Nanotechnology has used for alterations of the genetic structures of crop plants, thereby facilitating their improvement. Nanotechnology may offer in agronomic activities, with particular attention to critical features, challenging matters, and investigation needs for professional risk assessment and management in this developing field (Prasad et al. 2017). Nano-fertilizers (nano-coated fertilizers, nano-sized nutrients, or carbon-based nanomaterials or engineered metaloxide), and nano-pesticides (inorganic nanomaterials or nano-formulations of conventional active ingredients), may provide a targeted/controlled release of agrochemicals, aimed to obtain their fullest biological effectiveness without over-dosage (Iavicoli et al. 2017). Smart delivery of foods, a fast specimen of biological and chemical impurity, bioseparation of proteins and nano-encapsulation of nutritional supplements are some of the new areas of nanotechnology for food and agriculture (Sozer and Kokini 2009). Reduced biosynthesis of chlorophyll by magnetic nanoparticles of $\mathrm{Fe}_{3} \mathrm{O}_{4}$ induced a similar and statistically important decrease of chlorophyll and carotene levels of seedlings in sunflower (Ursache-Oprisan et al. 2011). The response of seedlings in Zea mays to the administration of the same range of $\mathrm{Fe}_{3} \mathrm{O}_{4}$ NPs concentration caused by the decrease of chlorophyll while the seedlings of Cucurbita pepo showed a minor elevation of chlorophyll contents (Racuciu et al. 2009). Thiruvengadam et al. (2015) reported that silver nanoparticles (AgNPs) could regulate the expression of genes involved in the metabolic pathways of carotenoids, phenolics, and glucosinolate in turnips. However, in addition to plants, nanomaterials can also affect animals, such as Eisenia fetida (earthworms), which evade AgNP-improved soil (ShoultsWilson et al. 2011).

Nano-sized calcium carbonate was prepared by reaction of sodium carbonate and calcium chloride by the reversedphase microemulsion technique and then loaded with the pesticide validamycin. It exhibited excellent germicidal activity against Rhizoctonia solani than validamycin later 7 days, and the time of the release of validamycin was prolonged to 2 weeks. The loading efficiency, stability, sustained-release performance and excellent ecological compatibility of the substance, the system for its use may be prolonged to another hydrophilic pesticide (Qian et al. 2011). Guan and Hubacek (2010) encapsulated the imidacloprid with a coating of chitosan and sodium alginate via layer-bylayer self-assembly, increasing its growth rate in soil applications. Moreover, as a vehicle for active materials (pesticides, fertilizers, or plant growth regulators), nanoparticles can also be synthesized through catalytic oxidation-reduction. Subsequent use of these materials would decrease the quantity of these active constituents in the environment and reduce the time through which the environment is exposed to the effects of the nanomaterials. Using nanotechnology to create new formulations has revealed significant potential in enlightening the efficiency and security of pesticides. The improvement of nano-based pesticide formulation aims at the complete release of necessary and adequate amounts of their active constituents in responding to environmental triggers and biological demands through controlled release mechanisms (Zhao et al. 2017). The nanoparticle-mediated transformation has the potential for genetic changes of plants for further development. The use of nanotechnology in plant pathology goals exact agricultural difficulties in plant-pathogen interactions and bring new ways for crop protection. Nair et al. (2010) studied the delivery of nanoparticulate materials to plants and their eventual effects, which could deliver some perceptions for the safe use of this novel technology for the improvement of crops. Some potential applications of nanoscale science, engineering, and nanotechnology for agriculture, expressly designed to improve and to protect agronomic yields and crop production as well as to detect and remediate environmental pollutants, have been addressed with attention focused on emerging occupational risks in this field (Iavicoli et al. 2017).

\section{Conclusions}

In conclusion, nanotechnology has become progressively important in the food industry. Food innovation is observed as one of the sector areas in which nanotechnology will play a major part in the forthcoming. New and future innovation is nanotechnology that has exceptionally extraordinary property in food source chain (precision farming techniques, smart feed, enhancement of food texture and quality, bioavailability/nutrient values, packaging, labeling, crop production and use of agrochemicals such as nano-pesticides, nano-fertilizers, and nano-herbicides) round the world agricultural sector. Nanofood packaging resources may widen nourishment life, upgrade food safety, prepared customers that food is sullied or destroyed, repair tears in packaging, and uniform release added substances to grow the life of 
the food in the package. To maintain leadership in food and food-processing industry, one must work with nanotechnology and nanobio-info in the future. The future belongs to new products and new processes with the goal to customize and personalize the products. Improving the safety and quality of food will be the first step. Finally, nanotechnology enables to change the existing food systems and processing to ensure products safety, creating a healthy food culture, and enhancing the nutritional quality of food.

Acknowledgements This paper was supported by the KU Research Professor Program of Konkuk University, Seoul, South Korea.

\section{Compliance with ethical standards}

Conflict of interest The authors have declared that there is no conflict of interest.

\section{References}

Alfadul SM, Elneshwy AA (2010) Use of nanotechnology in food processing, packaging and safety review. Afr J Food Agric Nutr Dev 10(6):2719-2739

Amaral DMF, Bhargava K (2015) Essential oil nanoemulsions and food applications. Adv Food Technol Nutr Sci Open J 1:84-87

Arévalo FJ, Granero AM, Fernández H, Raba J, Zón MA (2011) Citrinin (CIT) determination in rice samples using a micro fluidic electrochemical immunosensor. Talanta 83:966-973

Arshak K, Adley C, Moore E et al (2007) Characterization of polymer nanocomposite sensors for quantification of bacterial cultures. Sens Actuators B Chem 126:226-231

Ashwood P, Thompson R, Powell J (2007) Fine particles that adsorb lipopolysaccharide via bridging calcium cations may mimic bacterial pathogenicity towards cells. Exp Biol Med 232:107-117

Badgley C, Perfecto I (2007) Can organic agriculture feed the world. Renew Agric Food Syst 22:80-85

Bhattacharya S, Jang J, Yang L, Akin D, Bashir R (2007) Biomems and nanotechnology-based approaches for rapid detection of biological entities. J Rapid Methods Autom Microbiol 15:1-32

Biswal SK, Nayak AK, Parida UK, Nayak PL (2012) Applications of nanotechnology in agriculture and food sciences. Int J Inno Sci 2:21-36

Bratovčić A, Odobašić A, Ćatić S, Šestan I (2015) Application of polymer nanocomposite materials in food packaging. Croatian J Food Sci Technol 7:86-94

Canham LT (2007) Nanoscale semiconducting silicon as a nutritional food additive. Nanotechnology 18:185704

Cha D, Chinnan M (2004) Biopolymer-based antimicrobial packaging: a review. Crit Rev Food Sci Nutr 44:223-237

Chen H, Yada R (2011) Nanotechnologies in agriculture: new tools for sustainable development. Trends Food Sci Technol 22:585-594

Choi AJ, Kim CJ, Cho YJ, Hwang JK, Kim CT (2011) Characterization of capsaicin-loaded nano-emulsions stabilized with alginate and chitosan by self-assembly. Food Bioprocess Tech 4:1119-1126

Chung IM, Rajakumar G, Gomathi T et al (2017) Nanotechnology for human food: advances and perspective. Front Life Sci 10(1):63-72

Cushen M, Kerry J, Morris M et al (2012) Nanotechnologies in the food industry-recent developments, risks, and regulation. Trends Food Sci Technol 24:30-46
Davis D, Guo X, Musavi L et al (2013) Gold nanoparticle-modified carbon electrode biosensor for the detection of listeria monocytogenes. Ind Biotechnol 9:31-36

Ezhilarasi PN, Karthik P, Chhanwal N, Anandharamakrishnan C (2013) Nanoencapsulation techniques for food bioactive components: a review. Food Bioprocess Tech 6:628-647

Galarreta BC, Tabatabaei M, Guieu V, Peyrin E, Lagugne-Labarthet F (2013) Microfluidic channel with embedded SERS 2D platform for the aptamer detection of ochratoxin A. Anal Bioanaltical Chem 405:1613-1621

Giannakas A, Vlacha M, Salmas C et al (2016) Preparation, characterization, mechanical, barrier and antimicrobial properties of chitosan/PVOH/clay nanocomposites. Carbohydr Polym 140:408-415

Guan D, Hubacek K (2010) China can offer domestic emission capand-trade in post 2012. Environ Sci Technol 44:5327

Guo L, Feng J, Fang Z, Xu J, Lu X (2015) Application of microfluidic "lab-on-a-chip" for the detection of mycotoxins in foods. Trends Food Sci Technol 46:252-263

Gupta AK, Gupta M (2005) Synthesis and surface engineering of iron oxide nanoparticles for biomedical applications. Biomaterials 26:3995-4021

Gupta S, Moulik SP (2008) Biocompatible microemulsions and their prospective uses in drug delivery. J Pharm Sci 97:22-45

Gupta A, Eral HB, Hatton TA, Doyle PS (2016) Nanoemulsions: formation, properties, and applications. Soft Matter 12:2826-2841

Hervas M, Lopez MA, Escarpa A (2011) Integrated electrokinetic magnetic bead-based electrochemical immunoassay on microfluidic chips for reliable control of permitted levels of zearalenone in infant foods. Analyst 136:2131-2138

Honarvar Z, Hadian Z, Mashayekh M (2016) Nanocomposites in food packaging applications and their risk assessment for health. Electron Physician 8(6):2531-2538

Huang Q, Yu H, Ru Q (2010) Bioavailability and delivery of nutraceuticals using nanotechnology. J Food Sci 75:R50-R56

Iavicoli I, Leso V, Beezhold DH, Shvedova AA (2017) Nanotechnology in agriculture: opportunities, toxicological implications, and occupational risks. Toxicol Appl Pharmacol 329:96-111

Jain A, Ranjan S, Dasgupta N, Ramalingam C (2016) Nanomaterials in food and agriculture: an overview of their safety concerns and regulatory issues. Crit Rev Food Sci Nutr 6:1-21

Jebel FS, Almasi H (2016) Morphological, physical, antimicrobial and release properties of $\mathrm{ZnO}$ nanoparticles-loaded bacterial cellulose films. Carbohydr Polym 149:8-19

Joseph T, Morrison M (2006) Nanotechnology in agriculture and food. www.nanoforum.org

Kim H, Lee J, Kim JE et al (2013) Plum coatings of lemongrass oil-incorporating carnauba wax-based nanoemulsion. J Food Sci 78(10): 1551-1559

Komaiko JS, McClements DJ (2016) Formation of food-grade nanoemulsions using low-energy preparation methods: a review of available methods. Compr Rev Food Sci Food Saf 15:331

Kour H, Malik AA, Ahmad N et al (2015) Nanotechnology-new lifeline for the food industry. Crit Rev Food Sci Nutr 5:0. http s://doi.org/10.1080/10408398.2013.802662

Kuang DM, Peng C, Zhao Q et al (2010) Tumor-activated monocytes promote the expansion of IL-17-producing CD8+ T cells in hepatocellular carcinoma patients. J Immunol 185:1544-1549

Kuswandi B (2017) Environmental friendly food nano-packaging. Environ Chem Lett 15(2):205-221

Lee JS, Hong DY, Kim ES, Lee HG (2017) Improving the water solubility and antimicrobial activity of silymarin by nanoencapsulation. Colloids Surf B Biointerfaces 154:171-177

Lemes AP, Marcato PD, Ferreira OP, Alves OL, Duran N (2008) Nanocomposites of poly(3-hydroxybutyrate-co-3-hydroxyvalerate)

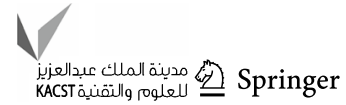


reinforced with carbon nanotubes and oxidized carbon nanotubes. Proc Nanotechnol Appl 615-085:72-75

Li Z, Sheng C (2014) Nanosensors for food safety. J Nanosci Nanotechnol 14(1):905-912

Liu HY, Lin SL, Chan SA, Lin TY, Fuh MR (2013) Microfluidic chipbased nano-liquid chromatography tandem mass spectrometry for quantification of aflatoxins in peanut products. Talanta 113:76

López-rubio A, Gavara R, Lagaron JM (2006) Bioactive packaging: turning foods into healthier foods through biomaterials. Trends Food Sci Technol 17:567-575

Martínez-Bueno MJ, Hernando MD, Uclés S et al (2017) Identification of non-intentionally added substances in food packaging nano films by gas and liquid chromatography coupled to orbitrap mass spectrometry. Talanta 172:68-77

McClements DJ, Rao J (2011) Food-grade nanoemulsions: formulation, fabrication, properties, performance, biological fate, and potential toxicity. Crit Rev Food Sci Nutr 51:285-330

Mihindukulasuriya SDF, Lim LT (2014) Nanotechnology development in food packaging: a review. Trends Food Sci Technol 40(2):149-167

Mills A, Hazafy D (2009) Nanocrystalline $\mathrm{SnO}_{2}$-based, UVB activated colorimetric oxygen indicator. Sens Actuators B Chem 136:344-349

Mousavi SR, Rezaei M (2011) Nanotechnology in agriculture and food production. J Appl Environ Biol Sci 1(10):414-419

Mozafari MR (2006) Bioactive entrapment and targeting using nanocarrier technologies: an introduction in nanocarrier technologies. In: Mozafari MR (ed) Frontiers of nanotherapy. Springer, The Netherlands, pp 1-16

Nair R, Varghese SH, Nair BG et al (2010) Nanoparticulate material delivery to plants. Plant Sci 179:154-163

Novo P, Moulasa G, Chua V, Condea JP (2012) Lab-on-chip prototype platform for ochratoxin a detection in wine and beer. Procedia Eng 47:550-553

Novo P, Moulas G, França Prazeres DM, Chu V, Conde JP (2013) Detection of ochratoxin $\mathrm{A}$ in wine and beer by chemiluminescence-based ELISA in microfluidics with integrated photodiodes. Sens Actuators B 176:232-240

Oca-Avalos JMM, Candal RJ, Herrera ML (2017) Nanoemulsions: stability and physical properties. Curr Opin Food Sci 16:1-6

Othman SH (2014) Bio-nanocomposite materials for food packaging applications: types of biopolymer and nano-sized filler. Agric Agric Sci Procedia 2:296-303

Pandey S, Zaidib MGH, Gururani SK (2013) Recent developments in clay-polymer nanocomposites. Sci J Rev 2:296-328

Parker CO, Lanyon YH, Manning M, Arrigan DWM, Tothill IE (2009) Electrochemical immunochip sensor for aflatoxin M1 detection. Anal Chem 81:5291

Pathakoti K, Manubolu M, Hwang H (2017) Nanostructures: current uses and future applications in food science. J Food Drug Anal 25(2):245-253

Pradhan N, Singh S, Ojha N et al (2015) Facets of nanotechnology as seen in food processing, packaging, and preservation industry. Biomed Res Int 365672:17

Prasad R, Bhattacharyya A, Nguyen QD (2017) Nanotechnology in sustainable agriculture: recent developments, challenges, and perspectives. Front Microbiol. https://doi.org/10.3389/fmicb.2017 .01014

Qian K, Shi T, Tang T et al (2011) Preparation and characterization of nano-sized calcium carbonate as controlled release pesticide carrier for validamycin against Rhizoctonia solani. Microchim Acta 173(1-2):51-57

Racuciu M, Creanga D, Olteanu Z (2009) Water based magnetic fluid impact on young plants is growing. Rom Rep Phys 61(2):259-268

Ravichandran R (2010) Nanotechnology applications in food and food processing: innovative green approaches, opportunities, and uncertainties for the global market. Int J Green Nanotechnol $1(2): 72-96$

Reynolds G (2007) FDA recommends nanotechnology research, but not labeled. Food Production Daily.com News, July 26, 2007

Rhim JW, Park HM, Ha CS (2013) Bio-nanocomposites for food packaging applications. Prog Polym Sci 38:1629-1652

Rivas GA, Miscoria SA, Desbrieres J, Berrera GD (2006) New biosensing platforms based on the layer-by-layer self-assembling polyelectrolytes on Nafion/carbon nanotubes-coated glassy carbon electrodes. Talanta 71:270-275

Sauceda-Friebe JC, Karsunke XYZ, Vazac S, Biselli S, Niessner R, Knopp D (2011) Regenerable immuno-biochip for screening ochratoxin A in green coffee extract using an automated microarray chip reader with chemiluminescence detection. Anal Chim Acta 689:234-242

Sekhon BS (2014) Nanotechnology in agrifood production: an overview. Nanotechnol Sci Appl 7:31-53

Shankar S, Rhim JW (2016) Polymer nanocomposites for food packaging applications. In: Dasari A, Njuguna J (eds) Functional and physical properties of polymer nanocomposites. Wiley, Chichester

Shelke NB, Vijay Kumar S, Mahadevan KM, Sherigara BS, Aminabhavi TM (2008) Synthesis, characterization, and evaluation of copolymers based on $\mathrm{N}$-isopropylacrylamide and 2-ethoxyethyl methacrylate for the controlled release of felodipine. J Appl Polymer Sci. 110:2211-2217

Shim WB, Dzantiev BB, Eremin SA, Chung DH (2009) One-step simultaneous immunochromatographic strip test for multianalysis of ochratoxin a and zearalenone. J Microbiol Biotechnol 19:83-92

Shoults-Wilson WA, Reinsch BC, Tsyusko OV et al (2011) Effect of silver nanoparticle surface coating on bioaccumulation and reproductive toxicity in earthworms (Eisenia fetida). Nanotoxicology $5: 432-444$

Silva HD, Cerqueira MA, Vicente AA (2012) Nanoemulsions for food applications: development and characterization. Food Bioprocess Tech 5:854-867

Singh N (2015) An overview of the prospective application of nanoemulsions in foodstuffs and food packaging. ASIO J Microbiol Food Sci Biotechnol Innova 1(1):20-25

Singh T, Shukla S, Kumar P et al (2017) Application of nanotechnology in food science: perception and overview. Front Microbiol. https://doi.org/10.3389/fmicb.2017.01501

Sonkaria S, Ahn SH, Khare V (2012) Nanotechnology and its impact on food and nutrition: a review. Recent Pat Food Nutr Agric $4: 8-18$

Sorrentino A, Gorrasi G, Vittoria V (2007) Potential perspectives of bio nanocomposites for food packaging applications. Trends Food Sci Technol 18:84-95

Sozer N, Kokini JL (2009) Nanotechnology and its applications in the food sector. Trends Biotechnol 27:82-89

Sugumar S, Singh S (2016) Nanoemulsion of orange oil with nonionic surfactant produced emulsion using ultrasonication technique: evaluating against food spoilage yeast. Appl Nanosci 6(1):113-120

Sun Y, Xia Z, Zheng J et al (2015) Nanoemulsion-based delivery systems for nutraceuticals: influence of carrier oil type on the bioavailability of pterostilbene. J Funct Foods 13:61-70

Tang D, Sauceda JC, Lin Z et al (2009) Magnetic nanogold microspheres-based lateral-flow immunodipstick for rapid detection of aflatoxin B2 in food. Biosens Bioelectron 25:514-518

Thiruvengadam M, Gurunathan S, Chung IM (2015) Physiological, metabolic, and transcriptional effects of biologically-synthesized silver nanoparticles in turnip (Brassica rapa $\mathrm{ssp}$. rapa L.). Protoplasma 252:1031-1046

Ursache-Oprisan M, Focanici E, Creanga D, Caltun O (2011) Sunflower chlorophyll levels after magnetic nanoparticle supply. Afr J Biotechnol 10(36):7092-7098 
Valdés MG, González ACV, Calzón JAG, Díaz-García ME (2009) Analytical nanotechnology for food analysis. Microchim Acta 166:1-19

Vidhyalakshmi R, Bhakyaraj R, Subhasree RS (2009) Encapsulation the future of probiotics-a review. Adv Biol Res 3:96-103

Vidotti M, Carvalhal RF, Mendes RK et al (2011) Biosensors based on gold nanostructures. J Braz Chem Soc 22:3-20

Wang X, Jiang Y, Wang YW et al (2008) Enhancing anti-inflammation activity of curcumin through $\mathrm{O} / \mathrm{W}$ nanoemulsions. Food Chem 108:419-424
Youssef AM (2013) Polymer nanocomposites as a new trend for packaging applications. Polym Plast Technol Eng 52(7):635-660

Zhao W, Lu J, Ma W et al (2011) Rapid on-site detection of Acidovorax avenae subsp. Citrulli by gold-labeled DNA strip sensor. Biosens Bioelectron 26:4241-4244

Zhao X, Cui H, Wang Y et al (2017) Development strategies and prospects of nano-based smart pesticide formulation. J Agric Food Chem. https://doi.org/10.1021/acs.jafc.7b02004 